\title{
Mentorship and workplace support needs of new graduate physiotherapists in rural and remote settings: A qualitative study
}

\author{
R. Martin ${ }^{1}$, A. Mandrusiak ${ }^{1}$, A. Lu $u^{2} \&$ R. Forbes ${ }^{1}$
}

\begin{abstract}
Introduction: New graduate physiotherapists working in rural and remote Australia must develop unique skills to work in this setting, while at the same time managing the difficulties typically faced by novice practitioners entering the workforce. Workplace mentorship is commonly used to facilitate this transition, yet little is known about new graduate physiotherapists' needs in rural and remote settings. This study aimed to explore workplace mentorship in rural and remote Australia from the perspective of new graduate physiotherapists and investigate what new graduates feel they need from mentorship that is specific to rural and remote settings.
\end{abstract}

Methods: A qualitative study with a thematic analytical approach was used. Semistructured interviews of 12 new graduate physiotherapists working in rural and remote settings were undertaken within the graduates' first 8 to 10 months of clinical practice. Interviews were audio-recorded between August and November 2019.

Results: Three key themes were constructed during the analysis: 1) Mentorship is best when it is frequent and consistent, 2) Initiating mentorship can be challenging and 3) Mentorship needs to be accessible and individualised.

Conclusion: There are challenges to mentorship of new graduate physiotherapists in rural and remote areas, including infrequency of mentorship, limited access to mentors and reluctance to request support. Whilst new graduate physiotherapists desire mentorship

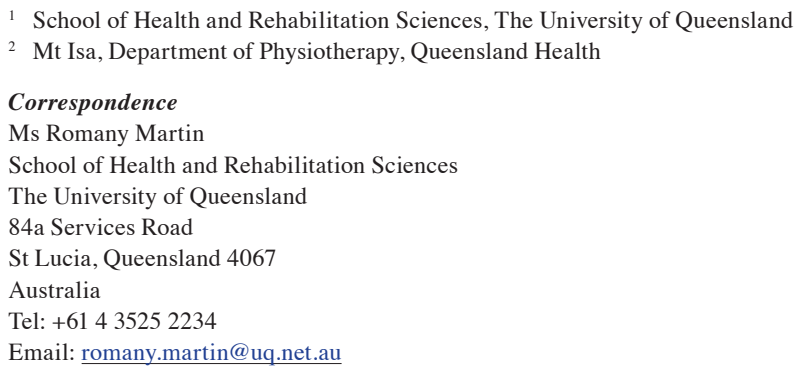


that is accessible, individualised and, ultimately, similar to what they perceive they would receive in the metropolitan setting, they acknowledge that to suit rural and remote localities, the delivery must be flexible. Mentorship is considered critical to support new graduates entering rural and remote settings.

Keywords: rural; remote; new graduate; physiotherapy; mentorship

\section{Introduction}

Entering the workforce and transitioning to independent practice is a process associated with feelings of stress and uncertainty (Ferguson, 2011; Foster-Seargeant, 2001). This is due to a significant increase in expectations (Davies et al., 2016) and the requirement to progress from a supervised student to an independent clinician who practises competently and safely in a clinical environment (Black et al., 2010). The availability and quality of supervision and support during the transition from student to new graduate is recognised as fundamental to sustainable and effective integration into the workplace (Ducat \& Kumar, 2015; Lea \& Cruickshank, 2015). Therefore, it is important to consider new graduates' experiences of supervision and support.

Australian rural and remote settings provide additional challenges for new graduates, such as a lack of professional supervision and mentorship (Lea \& Cruickshank, 2015). The Centre for Allied Health Evidence (2009) recommended that in rural and remote practice, support programs and mentorship should be provided in order to reduce the challenges presented by service delivery in these settings, including high workloads, diversity of clients and professional isolation. The review emphasised that a holistic approach, including adequate orientation, is needed to facilitate the transition to a rural locality and integration within the community (CFAHE, 2009).

Mentorship refers to the support of individuals in the professional setting and encompasses clinical supervision and professional development opportunities (Moran et al., 2014). New allied health graduates in rural settings face unique challenges, including a lack of mentoring and adequate supervision (CFAHE, 2009). Within the nursing profession, new graduates in rural and remote settings have reported that their mentorship needs went largely unmet (Lea \& Cruickshank, 2016), and instead, they experienced a lack of structured support (Hegney et al., 2013). This impacts transitional experiences and the subsequent attrition of graduates within the rural practice environment (Ross et al., 2019; Spiers \& Harris, 2015). A significant barrier to mentorship amongst rural pharmacists is the lack of staff to facilitate training and support programs (Leversha et al., 2001). This is also reflected in the findings of Moran et al. (2014), who identified that access to adequate human resources to facilitate support programs is linked to successful outcomes for new allied health graduates. Within the larger allied health workforce of rural and remote 
Australia, there is an identified lack of supervision and mentors, especially within a new graduate's own discipline (Devine, 2006). Ultimately, clinical supervision in rural and remote allied health has many unique characteristics, including, but not limited to, drawing on professional support from the multidisciplinary team and a limited pool of eligible mentors (Martin et al., 2019). These factors may exacerbate the challenges of transitioning to independent practice (Foster-Seargeant, 2001; Stoikov et al., 2020).

Upon entering the workforce, new graduate physiotherapists in rural and remote practice are expected to work across a broad scope (CFAHE, 2009). New graduate physiotherapists in these settings must also be ready to care for patients with complex conditions (CFAHE, 2009) and meet competency requirements across clinical, administrative and professional domains (NRHA, 2004). Additional to this, physiotherapists in rural and remote Australia often practise as sole practitioners, compounding these challenges (CFAHE, 2009). When new graduates have limited exposure to experienced physiotherapists, it significantly decreases the availability and quality of supervision and mentorship (Lea \& Cruickshank, 2015), and this must be considered when planning new graduate support. When effective professional supervision is implemented in rural and remote allied health services, it may enhance professional enthusiasm, knowledge, confidence and patient safety, and reduce isolation (Ducat et al., 2016).

With this in mind, strategies to create a positive supervision culture (Ducat et al., 2016) that enable support and mentorship of all new graduates are necessary (Davies et al., 2016). Investigating the mentorship needs of new graduate physiotherapists in rural and remote Australia will provide insight for higher education providers, employers and the profession to facilitate preparedness for independent clinical practice.

Therefore, the aims of this study are to: 1) investigate the experience of workplace mentorship in rural and remote Australia from the perspective of new graduate physiotherapists and 2) explore the specific mentorship needs of new graduate physiotherapists working in rural and remote Australia.

\section{Methods}

A qualitative design was undertaken using semi-structured thematic interviews (Kvale, 1996) of new graduate physiotherapists working in rural or remote Queensland, Australia. A semi-structured interview framework was developed (Figure 1) by the research team and was influenced by similar interview frameworks used within the literature (Williams et al., 2016), the aims of the research and the clinical expertise of the authors. Ethical clearance was obtained from the University of Queensland-Institutional Human Research Ethics, approval number 2019001696. 
MENTORSHIP AND WORKPLACE SUPPORT NEEDS

\section{Figure 1}

The Semi-Structured Interview Guide

\section{Interview framework}

1. Can you tell me about your workplace and your typical workday?

2. Can you tell me about some of the challenges that you face working in a rural setting?

a. What makes that challenging?

b. What do you do to manage that?

3. What type of mentoring or support do you get?

4. Who decided what type of mentoring you get?

5. Who decides what topics you cover?

6. What do you think you need from mentoring?

To be included in the study, new graduates had to have been working as a physiotherapist in a rural setting, as defined by the Modified Monash Model classifications (MM3 through to MM7) (Australian Bureau of Statistics, 2019) for a minimum of 8 weeks. Eight weeks was chosen to ensure that the participants had adequate experience to reflect on. New graduates were also required to have graduated from an entry-level physiotherapy degree in the 2 years prior to participation, to maintain the definition of a new graduate (Chipchase et al., 2008).

New graduate physiotherapists were recruited through snowball sampling via contacts of the research team. The contacts were physiotherapists employed in rural or remote settings. The professional contacts approached their colleagues in person and requested a binary yes-no consent to receive further contact for this study. Those who consented to be contacted were then emailed further information regarding the study. Purposeful sampling was used to ensure that the study included a range of participants (Patton, 2002).

After informed consent, a member of the research team who was trained in interviewing (RM) undertook the telephone interviews using a semi-structured interview framework (Figure 1). These interviews were arranged based on the participants' availability. The interviews were audio-recorded between August and November 2019 and transcribed verbatim. Transcription and analysis of data were undertaken on a continual basis until thematic sufficiency was achieved. Thematic sufficiency was determined when no new themes were interpreted during the analysis of three additional transcripts and when the constructed themes aligned with the research aims (Varpio, Ajjawi, et al., 2017). Data was de-identified to ensure participant confidentially.

Data was analysed using thematic analysis (Braun \& Clarke, 2006), which is part of the descriptive phenomenological method (Englander, 2016). The research team aimed to 


\section{MENTORSHIP AND WORKPLACE SUPPORT NEEDS}

construct the "probable truth" arising from the data, based on the systemically gathered experiences and perspectives of the participants (Bunniss \& Kelly, 2010). A subjectivist inductive approach was undertaken to explore the phenomenon, as the researchers explored the meanings constructed by individual participants (Varpio, Paradis, et al., 2020). The lead researcher (RM) immersed themself in the data to become familiar with the experiences and perceptions voiced by the participants. Initial codes and illustrative participant quotes were collated. These codes and quotes were then constructed into themes. Analysis was independently completed by two reviewers, and consensus of themes was presented to the research team for discussion and additional detail acquisition. Differences between coders were discussed openly until consensus was reached. Themes were refined with reference to the transcripts until reviewer consensus was reached. Qualitative data management software NVivo10 (QSR International Pty Ltd, 2018) was used throughout the analysis process.

Throughout the analysis process, efforts were made to maximise rigour and to ensure trustworthiness, credibility and transparency (Long \& Johnson, 2000; Patton, 2002). This included ensuring that the semi-structured interview framework was adhered to for all interviews and all interviews were audiotaped and a secondary review of the recordings was conducted post transcription.

Actions to ensure the reflexivity of the analysis process were undertaken, as the descriptive phenomenological method requires researchers to play an active role in the construction of the results, which may affect the knowledge produced from the work (Carpenter $\&$ Suto, 2006). Prior to analysis, the lead researcher (RM) undertook the process of epoche to identify any preestablished knowledge regarding the subject matter (Moustakas, 1994). This allowed the researcher to intentionally study the data, rather than the authors' experiences being an antecedent for the results (Englander, 2016). The perspectives, characteristics and experiences of both researchers who undertook analysis of the data were examined to ensure the transparency of the themes constructed (Varpio, Ajjawi, et al., 2017). The lead researcher (RM) has been influenced by the body of literature regarding rural health and rural mentorship and has personal experiences of being mentored as a physiotherapist. The author who undertook the secondary analysis (RF) has significant experience supervising physiotherapy students and graduates through her role as a musculoskeletal physiotherapist and is a physiotherapy academic staff member. These factors were considered continually throughout the analysis process. 


\section{Table 1}

Demographic Details of Sample

\begin{tabular}{l|c}
\hline Demographic & n (\%) \\
\hline Age (years) & $\mathbf{2 3 . 3 3 ~ ( r a n g e ~ 2 1 - 3 0 ) ~}$ \\
\hline Gender & \\
$\quad$ Female & $8(66.7 \%)$ \\
Male & $4(33.3 \%)$ \\
\hline Physiotherapy degree & \\
Bachelor entry & $8(66.7 \%)$ \\
Masters entry & $4(33.3 \%)$ \\
\hline Rural location (Modified Monash Model) & \\
Large rural towns (MM3) & $2(16.7 \%)$ \\
Medium rural towns (MM4) & $6(50 \%)$ \\
Small rural towns (MM5) & $0(0 \%)$ \\
Remote communities (MM6) & $0(0 \%)$ \\
Very remote communities (MM7) & $4(33.3 \%)$ \\
\hline
\end{tabular}

\section{Table 2}

Demographic Details of Participants

\begin{tabular}{c|c|l|l|l|c}
\hline $\begin{array}{c}\text { Participant } \\
\text { Reference }\end{array}$ & Age & Gender & $\begin{array}{c}\text { Graduate } \\
\text { Level }\end{array}$ & Discipline of Work & $\begin{array}{c}\text { Modified Monash } \\
\text { Model Scale }\end{array}$ \\
\hline 1 & 30 & Female & Masters & Outpatients/inpatients & 4 \\
\hline 2 & 21 & Female & Bachelor & Outpatients/inpatients & 4 \\
\hline 3 & 23 & Female & Bachelor & Outpatients/inpatients & 7 \\
\hline 5 & 22 & Female & Bachelor & Outpatients & 4 \\
\hline 6 & 22 & Female & Bachelor & Outpatients & 4 \\
\hline 7 & 23 & Male & Bachelor & Outpatients & 3 \\
\hline 9 & 26 & Male & Masters & Outpatients & 7 \\
\hline 10 & 23 & Male & Masters & Outpatients/inpatients & 7 \\
\hline 11 & 23 & Male & Masters & Inpatients & 3 \\
\hline 12 & 22 & Female & Bachelor & Outpatients/inpatients & 7 \\
\hline
\end{tabular}




\section{MENTORSHIP AND WORKPLACE SUPPORT NEEDS}

\section{Results}

Fifteen participants were recruited and all consented to be interviewed for the study. Three participants failed to meet the remoteness scale as determined by the Modified Monash Model and were excluded from the sample. All interviews were completed using the semi-

\section{Table 3}

\section{Further Illustrative Quotes}

\section{Theme 1: Mentorship is best when it is frequent and consistent.}

I probably would have benefited from a bit more mentoring. (Participant 10)

No, I wasn't offered any. So, we're meant to get like an hour clinical supervision a week. I didn't even really get offered it whilst I was there. (Participant 2)

I think new grads are supposed to have... like feedback and kind of like discussion time with the supervisors, where they can ask any questions and go through certain things, so that was offered to me pretty much every week, so that was really good. (Participant 6)

They also did these awesome like, new grad in-service slash check ins via telehealth. (Participant 5)

Being a new grad and being in a rural area where you are seeing sort of a bit of everything but then also working on your own. (Participant 2)

\section{Theme 2: Initiating mentorship can be challenging.}

If you have to go to the effort of writing an email or making the choice of picking up the phone ... you're already ... rethinking it so many times in your head, and I think it makes you go "Oh, is this actually a question worth asking?" or "Should I already know this?" or "Is this dumb?" (Participant 10) If I had like an urgent inquiry, I could call Townsville, but again, I didn't really know how to call. (Participant 2)

It was just finding out who to contact and who to go to ... I had to search for these people. (Participant 12)

I made the recommendation that maybe once a week, there could be a telephone call back to one of the outpatient physios ... but make it compulsory once a week, rather than, "if you need to call, call". (Participant 12)

\section{Theme 3: Mentorship needs to be accessible and individualised.}

Each week the new grads back in the [urban centre] were getting an inservice on a Tuesday afternoon. ... I think it would have been really nice if we, like, could have been Skyped back into that one, even though we weren't there. (Participant 11)

Once a week ... we have 2 hours of what's called supervision or mentoring essentially, and I have opportunity ... to discuss things across the week that I came across that were interesting, or I felt I had difficulty ... so that's been good for me. (Participant 7) 
MENTORSHIP AND WORKPLACE SUPPORT NEEDS

structured interview framework (Figure 1) and lasted 20-30 minutes (mean $=28$ minutes). Demographic data is outlined in Tables 1 and 2. Three key themes were generated from the analysis: 1) Mentorship is best when it is frequent and consistent, 2) Initiating mentorship can be challenging and 3) Mentorship needs to be accessible and individualised. Additional illustrative quotes are provided in Table 3.

\section{Theme 1: Mentorship is best when it is frequent and consistent}

Participants expressed their perception that mentorship in rural and remote settings varied in approach, accessibility and frequency. New graduates described "structured" mentorship as allocated time set aside for mentorship, with "unstructured" referring to casual conversations with mentors throughout the workday. The formality of mentorship also varied, with new graduates referring to formal mentorship that occurred around predetermined topics and informal mentorship in which the mentee led the discussion. The duration and frequency of mentorship was reported to be incongruent with their expectations of new graduate support, with some participants reportedly receiving no mentorship.

I suppose you're really on your own, you don't have that much support ... I had a phone number for the physio if needed, but it's not like I'm having any training or someone always there checking on me. (Participant 3)

Compounding the perceived lack of mentorship was limited exposure to physiotherapy colleagues and health professionals of other disciplines generally. Participants reflected that they were often a sole practitioner due to senior position vacancies and recruitment challenges, which resulted in feelings of stress and being overwhelmed. New graduates also perceived an increased sense of isolation resulting from minimal encounters with other clinicians and health professionals. In response to decreased staff resources, participants reported that they would often seek clinical support through other avenues, including their social circles and other professionals. One participant reported that they "primarily would speak to the occupational therapist" (Participant 9) for clinical discussion and feedback.

I definitely feel less supported here ... and sometimes I feel terrified, like I feel more at risk? Like I'm more liable here? (Participant 4)

Along with a variety of approaches to mentorship, new graduates reported that more frequent interactions with their mentors would have decreased the associated stress of transitioning into rural and remote practice.

I think I wouldn't leave a new grad alone for like, 3 days of the week, not having a senior there to ask questions. I think they do need someone there with them. (Participant 11) 


\section{MENTORSHIP AND WORKPLACE SUPPORT NEEDS}

\section{Theme 2: Initiating mentorship can be challenging}

New graduates often reported an awareness of support in rural and remote areas but also barriers and challenges related to initiating this support, including the need to actively seek support, a perceived lack of knowledge about how to access structured mentorship and a reluctance to prompt unstructured mentorship.

I think that the support out there is better than what people think, and if you are active in seeking it then you will not have a problem at all. (Participant 5)

Whilst new graduates were aware that they had to actively seek support, they felt they weren't equipped with the skills required to access unstructured support in rural or remote settings, especially where there are few local mentors. One participant shared their experience of working in a rural location as a sole practitioner and stated that being a sole practitioner significantly hindered access to mentorship. They also perceived challenges in creating networks with other professions in a new town, which compounded this difficulty.

I found ... that trying to link in with other people was quite difficult to do as well when I was brand new to the area ... and didn't really have those connections.... I think that's definitely like a difficulty. (Participant 2)

Participants described reluctance to initiate unstructured mentorship, as they did not want to be viewed as incompetent in their new workplace. New graduates felt that requesting time for mentorship was not a priority and that time in mentorship was taking away time allocated to patients, which often resulted in feelings of stress when requesting support. Participants acknowledged that this was mitigated by structured mentorship.

As a new graduate, I don't want to be seen as being incompetent, so I don't want to be seen as regularly calling if I have questions. Whereas I think if it was scheduled and an expectation that you do this phone call, it would be less daunting. (Participant 12)

Accessibility of mentorship was important to new graduates, received either in person or via digital means, such as via telephone, videoconference or email. Videoconferencing with senior staff at urban centres was suggested as a possibility for formal mentoring, however this presented its own challenges with scheduling between health services.

We did have a new graduate education program going on at [rural town] where I could teleconference in. But because of the way outreach worked, I was never able to teleconference in. (Participant 9) 


\section{MENTORSHIP AND WORKPLACE SUPPORT NEEDS}

\section{Theme 3: Mentorship needs to be accessible and individualised}

Local support was the preferred means of mentorship, with participants reporting that inperson mentorship was less daunting and enabled better outcomes. Local support was also perceived as more comprehensive, as participants were able to access their mentors for both structured and unstructured mentorship.

Yeah, I felt like I could ask him anything. He was really good, and even if I was a bit nervous or thought that I had done the wrong thing ... it's so much easier to ask a question that you're nervous about if the person is right there? It's preferable to have someone, just in the same room or .... in the same setting, that you can talk to about it and you can actually feel safe asking that question. (Participant 10)

Irrespective of how mentorship was engaged with, being able to access it was perceived as an expectation of new graduate practice regardless of location. Participants expressed a desire for continued learning and placed importance on mentorship as part of professional development.

I think new grads are supposed to have ... feedback and kind of like discussion time with supervisors where they can ask any questions and go through certain things, so that was offered to me pretty much every week, so that was really good. (Participant 6)

Most prominently, new graduates expressed that they desired mentorship that was individualised to their learning style and perceived needs. Participants acknowledged that they desired both structured and unstructured mentorship, despite its limited availability in rural and remote locations.

I think I would have wanted it just from the perspective of talking through my clients ... I did do that informally, but I didn't have a dedicated time to sit down and really go through it. (Participant 9)

Ultimately, new graduates reported that they desired similar mentorship content and a similar approach to what they perceived they would receive in the metropolitan setting, however they acknowledged that this would need to be delivered in different ways. Participants acknowledged that frequent mentorship was often not practical in rural and remote locations due to staffing constraints, smaller team size and fewer opportunities to interact with other clinicians, however they considered mentorship via telecommunications to be a satisfactory and viable option. Participants were surprised by the contrasts between support in urban and rural or remote settings. 
So in [metropolitan town] I have like, a "buddy" who ... I meet up with once a week to go over any complex cases...I can either shadow them or they can shadow me ... and then give me some feedback... There's none of that in [rural town] because, like I said, when I'm at the hospital, the other physio is out on outreach. (Participant 12)

One participant noted the perceived "stigma" regarding requesting mentorship in rural and remote workplaces and described that the culture of independent practice without interprofessional support was in stark contrast to the clinical supervision expected in the urban setting. Participants in rural and remote settings desired an environment where requesting mentorship was the norm, rather than feeling that this was an admission of incompetence.

It can be just the same out there, like it's an expectation you do it in [metropolitan city], so why not make it an expectation out rurally as well. (Participant 12)

\section{Discussion}

Overall, this study supports the perceived value of mentorship for new graduates and demonstrates that there are unique challenges to mentorship in the rural and remote setting, including infrequency of mentorship, limited access to mentors and reluctance to request support. New graduate physiotherapists desire mentorship that is accessible, individualised and, ultimately, aligned with their perceptions of what they would receive in the metropolitan setting. They acknowledge that the delivery must be flexible to suit rural and remote localities.

New graduates expressed that mentorship in the rural and remote setting was typically achieved through a series of interactions with different professionals, rather than one mentor. This is reflective of literature by Martin et al. (2019), who reported that new allied health graduates in rural Australia receive professional support from the multidisciplinary team. This is partly attributed to limited opportunities to engage with other physiotherapy colleagues in the rural setting as a result of smaller teams with large demand and staff resource constraints (CFAHE, 2009). New graduate members of the broader allied health profession subsequently have fewer opportunities for mentorship from members of their own profession (Devine, 2006). This is reported to impact the student-to-clinician transition into the workforce and, consequently, the ability to develop clinical and professional skills to deliver effective patient care (Wainwright et al., 2011).

New graduates in our study reported that support was not forthcoming in the transition to rural and remote clinical practice despite their desire for both clinical and professional guidance, which is a challenge also reported by new graduate nurses (Lea \& Cruickshank, 2016). New graduates felt that the unestablished networks for accessing mentorship were compounded by inadequate staffing, which is consistent with previous research (Calleja et 
al., 2019). Further, busy timetables of senior staff were perceived as a barrier to seeking mentorship, which is a challenge common to other rural and remote health disciplines (Lea \& Cruickshank, 2007). New graduates in this study desired more structured mentorship to alleviate the challenges of clinical practice, which is a critical finding, as sufficient mentorship is known to influence a new graduate's intention to remain employed in the setting (Tourangeau et al., 2010).

New graduates desired a protected time to engage in structured mentorship, which is a perception shared with new graduate nursing staff, who wish for quarantined time to sit with someone to set goals and discuss progress (Duchscher, 2008). This was attributed to the reluctance of new graduates to acknowldge that they required assistance. The mentor-led facilitation of a mentoring program has been recognised as a strategy for the successful implementation of new graduate support amongst multiple new graduate allied health professionals (Moran et al., 2014). New graduate practice is often reported as a hypersensitive and self-critical state (Duchscher, 2008), and this must be considered when designing mentorship programs. If accessing mentorship is perceived as an "admittance of incompetence", then the services are unlikely to be utilised. However, if it becomes an expectation, then mentorship may assist the facilitation of sustainable and effective integration into the workplace (Ducat \& Kumar, 2015; Lea \& Cruickshank, 2015).

In response to these challenges, new graduates identified facilitators to help achieve timely and relevant mentorship, including teleconferences, phone calls, emails with local staff and networking with practitioners in more urban settings. Mentorship programs utilising these technologies have long been implemented and result in self-reported improvements in confidence, knowledge and skill (Xavier et al., 2007). Amongst New Zealand midwives, mentoring via telecommunications has been identified as a viable option to remove the barrier of location and allow for a broader range of possible mentors (Stewart \& Wootton, 2005). This is important considering that a good mentee-mentor fit is a key factor associated with effective professional supervision arrangements (Ducat et al., 2016). Considering this, it is paramount that the technologies available to new graduates are coupled with appropriate training to facilitate uptake.

As expected, the specific mentorship content perceived by rural and remote new graduates to be most relevant differed significantly based on the individual's needs and clinical demands. An overarching emphasis was placed on mentorship content being relevant to the broad scope required in rural practice (CFAHE, 2009) and timely, albeit via any means; both of which are characteristics of effective mentorship (Ferguson, 2011). Research in other health professions highlights the need for mentorship that includes emotional support in order to address both professional and locality challenges (Lea \& Cruickshank, 2016). These findings are in alignment with Proctor's model of clinical supervision, which 


\section{MENTORSHIP AND WORKPLACE SUPPORT NEEDS}

focuses on three areas of supervision: normative (core mandatory training), formative (professional development and self-reflection) and restorative (supportive aspect for personal development) (Proctor, 1987). This model encompasses a critical understanding of mentorship in rural and remote locations and is reflective of the belief that mentorship needs to be proactive to suit the needs of the new graduate and inclusive of the personal, professional and clinical aspects of rural and remote practice (Ducat \& Kumar, 2015).

\section{Implications}

This study has identified that new graduate physiotherapists perceive difficulty accessing mentoring in rural and remote Australia and that they perceive ideal mentoring in the setting to be frequent, accessible and tailored to the individual needs of the graduate. This knowledge, along with the body of literature demonstrating the importance of mentorship in rural and remote settings, should encourage rural and remote workplaces to incentivise the implementation and evaluation of mentorship programs for new graduates. Further recommendations on strategies to overcome the challenges that have been raised by new graduate physiotherapists in our study are provided in Figure 2.

\section{Figure 2}

\section{Recommendations for practice}

Institutional-level recommendations:

- Incentivise the implementation and evaluation of mentorship programs.

- Provide human resources and education resources for effective and sustainable mentorship.

- Further research to investigate the experiences and perspective of stakeholders.

Educator-level recommendations:

- Facilitate access to mentorship (connection of appropriate mentors to mentees).

- Provide resources within the clinical environment for mentors:

- Dedicated time during work hours

- Education of senior staff in adapting teaching styles

- Planning learning activities and support strategies.

Resources within the clinical environment should be made available to senior staff and other mentors to facilitate new graduate transition to the workplace (Yoon et al., 2017). The practical implications of providing these resources must also be considered to ensure new graduate support strategies are in place, for example, facilitating dedicated time during work hours for self-directed new graduate professional development and educating senior staff to adapt teaching styles and plan learning activities to ensure new graduate support strategies are in place (CFAHE, 2009). To facilitate access to mentorship, assistance from 
line managers may facilitate the connection of appropriate mentors to mentees. These mentors may either be within the local organisation, from another rural equivalent or from metropolitan centres for more specific specialist clinical areas and accessed via e-mentoring, as demonstrated by Stewart and Wootton (2005). It should be acknowledged that an appropriate mix of all three may help address operational, geographical and clinical challenges. In alignment with Moran et al. (2014), human resource considerations must be provided in addition to the educational resources specific to the learning needs of the new graduate so that effective and sustainable mentorship can be delivered.

Further research is required within the physiotherapy setting to investigate the experiences and perspective of the other stakeholders involved in mentorship of new graduates, such as employers, mentors and universities. Finally, further research is warranted to evaluate structured and sustainable mentorship in alignment with the mentoring interventions outlined by Moran et al. (2014). This would enable the evaluation of whether structured mentorship programs within physiotherapy could align mentor and mentee expectations and facilitate pathways to access timely and appropriate support in rural and remote settings, commensurate to experience.

\section{Limitations}

As a result of previous training at a tertiary institution, the lead researcher was known to some participants, which is a limitation mitigated by the completion of a second independent analysis. This study has explored workplace mentorship from the single perspective of the mentee, excluding that of other stakeholders, such as the mentor, colleagues, supervisors, patients and policy makers, which may limit a complete understanding of mentorship for new graduate physiotherapists in rural and remote settings. Other limitations are present in the sample, with recruitment of participants restricted to a single state within Australia. The sample demonstrates an inequitable spread of rural locality, with no participants featured from small rural towns or remote communities. Without this limitation, the study may have arrived at different conclusions regarding mentorship of rural new graduates. Finally, to some in the field of health professional education, mentorship and clinical supervision are separate, although this paper does not distinguish between the two.

\section{Conclusion}

Overall, this study demonstrates that there are significant challenges to mentorship of new graduate physiotherapists in rural and remote areas, including infrequency of mentorship, limited access to mentors and reluctance to request support. New graduate physiotherapists desire mentorship that is equitable to what they perceive they would receive in the metropolitan setting, as well as mentorship that is accessible and individualised. 
MENTORSHIP AND WORKPLACE SUPPORT NEEDS

\section{Funding and conflicts of interest}

The authors have no conflicts of interest to declare. No funding was received for this project.

\section{References}

Australian Bureau of Statistics. (2019). Modified Monash Model. https://www.health.gov.au/ sites/default/files/download-pdf_0.pdf

Black, L., Jensen, G. M., Mostrom, E., Perkins, J., Ritzline, P. D., Hayward, L., \& Blackmer, B. (2010). The first year of practice: An investigation of the professional learning and development of promising novice physical therapists. Physical Therapy, 90(12), 1758-1773. https://doi.org/10.2522/ptj.20100078

Braun, V., \& Clarke, C. (2006). Using thematic analysis in psychology. Qualitative Research in Psychology, 3(2), 77-101. https://doi.org/10.1191/1478088706qp063oa

Bunniss, S., \& Kelly, D. R. (2010). Research paradigms in medical education research. Medical Education, 44(4), 358-366. https://doi.org/10.1111/j.1365-2923.2009.03611.x

Calleja, P., Adoteng-Kissi, B., \& Remero, B. (2019). Transition support for new graduate nurses to rural and remote practice: A scoping review. Nurse Education Today, 76, 8-20. https://doi.org/10.1016/j.nedt.2019.01.022

Carpenter, C., \& Suto, M. (2006). Qualitative research for occupational and physical therapists: A practical guide. Wiley Blackwell.

Centre for Allied Health Evidence (CFAHE). (2009). Literature review supporting the transition of allied health professionals to remote and rural practice. https://sarrah. org.au/sites/default/files/docs/appendix a literature review.pdf

Chipchase, L., Williams, M., \& Robertson, V. (2008). Preparedness of new graduate Australian physiotherapists in the use of electrophysical agents. Physiotherapy, 94(4), 274-280. https://doi.org/10.1016/j.physio.2008.09.003

Davies, J., Edgar, S., \& Debenham, J. (2016). A qualitative exploration of the factors influencing the job satisfaction and career development of physiotherapists in private practice. Manual Therapy, 25, 56-61. https://doi.org/10.1016/j.math.2016.06.001

Devine, S. (2006). Perceptions of occupational therapists practising in rural Australia: A graduate perspective. Australian Occupational Therapy Journal, 53(3), 205-210. https://doi.org/10.1111/j.1440-1630.2006.00561.x 


\section{MENTORSHIP AND WORKPLACE SUPPORT NEEDS}

Ducat, W., Martin, P., Kumar, S., Burge, V., \& Abernathy, L. (2016). Oceans apart, yet connected: Findings from a qualitative study on professional supervision in rural and remote allied health services. Australian Journal of Rural Health, 24(1), 29-35. https:// doi.org/10.1111/ajr.12192

Ducat, W., \& Kumar, S. (2015). A systematic review of professional supervision experiences and effects for allied health practitioners working in non-metropolitan health care settings. Journal of Multidiscipline Healthcare, 8, 397-407. https://doi.org/10.2147/ JMDH.S84557

Duchscher, J. E. B. (2008). A process of becoming: The stages of new nursing graduate professional role transition. Journal of Continuing Education in Nursing, 39(10), 441-450. https://doi.org/10.3928/00220124-20081001-03

Englander, M. (2016). The phenomenological method in qualitative psychology and psychiatry. International Journal of Qualitative Student Health Well-being, 11(1), Article 30682. https://doi.org/10.3402/qhw.v11.30682

Ferguson, L. M. (2011). From the perspective of new nurses: What do effective mentors look like in practice? Nurse Education in Practice, 11(2), 119-123. https://doi. org/10.1016/j.nepr.2010.11.003

Foster-Seargeant, E. (2001). The lived experience of new graduate physiotherapists in the first year of practice: Mentorship and program management [Master's dissertation, Lakehead University]. Lakehead University Library. https://knowledgecommons. lakeheadu.ca/handle/2453/3175

Hegney, D., Eley, R., \& Francis, K. (2013). Queensland nursing staffs' perceptions of the preparation for practice of registered and enrolled nurses. Nurse Education Today, 33(10), 1148-1152. https://doi.org/10.1016/j.nedt.2012.11.023

Kvale, S. (1996). An introduction to qualitative research interviewing. Sage.

Lea, J., \& Cruickshank, M. (2007). The experience of new graduate nurses in rural practice in New South Wales. Rural Remote Health, 7(4), Article 814. https://www.rrh.org.au/ public/assets/article documents/article print 814.pdf

Lea, J., \& Cruickshank, M. (2015). Supporting new graduate nurses making the transition to rural nursing practice: Views from experienced rural nurses. Journal of Clinical Nursing, 24(19-20), 2826-2834. https://doi.org/10.1111/jocn.12890 


\section{MENTORSHIP AND WORKPLACE SUPPORT NEEDS}

Lea, J., \& Cruickshank, M. (2016). The role of rural nurse managers in supporting new graduate nurses in rural practice. Journal of Nursing Management, 25(3), 176-183. https://doi.org/10.1111/jonm.12453

Leversha, A., Strasser, R., \& Teed, A. (2001). Training and support program for pharmacists in rural Victoria. Australian Journal of Rural Health, 9(1), 7-11. https://doi. org/10.1046/j.1440-1584.2001.00322.x

Long, T., \& Johnson, M. (2000). Rigour, reliability and validity in qualitative research. Clinical Effectiveness in Nursing, 4(1), 30-37. https://doi.org/10.1054/cein.2000.0106

Martin, P., Kumar, S., Lizarondo, L., \& Baldock, K. (2019). Debriefing about the challenges of working in a remote area: A qualitative study of Australian allied health professionals' perspectives on clinical supervision. PLOS One, 14(3), Article e0213613. https://doi.org/10.1371/journal.pone.0213613

Moran, A., Coyle, J., Pope, R., Boxall, D., Nancarrow, S. A, \& Young, J. (2014). Supervision, support and mentoring interventions for health practitioners in rural and remote contexts: An integrative review and thematic synthesis of the literature to identify mechanisms for successful outcomes. Human Resources for Health, 12, Article 10. https://doi.org/10.1186/1478-4491-12-10

Moustakas, C. (1994). Phenomenological research methods. Sage.

National Rural Health Alliance (NRHA). (2004). Under pressure and undervalued: Allied health professionals in rural and remote Australia. https://www.ruralhealth.org.au/ sites/default/files/position-papers/position-paper-04-11-05.pdf

Patton, M. (2002). Qualitative research and evaluation methods (3rd ed). Sage.

Proctor, B. (1987). Supervision: A co-operative exercise in accountability. In M. Marken \& M. Payn (Eds.), Enabling and ensuring: Supervision in practice (pp. 21-23). National Youth Bureau and the Council for Education and Training in Youth and Community Work.

Ross, B. M., Cameron, E., \& Greenwood, D. (2019). A qualitative investigation of the experiences of students and preceptors taking part in remote and rural community experiential placements during early medical training. Journal of Medical Education and Curricular Development, 6. https://doi.org/10.1177/2382120519859311

Spiers, M. C., \& Harris, M. (2015). Challenges to student transition in allied health undergraduate education in the Australian rural and remote context: A synthesis of barriers and enablers. Rural Remote Health, 15(2), Article 3069. https://doi. org/10.22605/RRH3069 


\section{MENTORSHIP AND WORKPLACE SUPPORT NEEDS}

Stewart, S., \& Wootton, R. (2005). A survey of e-mentoring among New Zealand midwives. Journal of Telemedicine and Telecare, 11(Suppl. 2), 90-92. https://doi. org/10.1258/135763305775124731

Stoikov, S., Maxwell, L., Butler, J., Shardlow, K., Gooding, M., \& Kuys, S. (2020). The transition from physiotherapy student to new graduate: Are they prepared? Physiotherapy Theory and Practice, 1-11. https://doi.org/10.1080/09593985.2020.1 744206

Tourangeau, A. E., Cummings, G., Cranley, L. A, Ferron, E. M., \& Harvey, S. (2010). Determinants of hospital nurse intention to remain employed: Broadening our understanding. Journal of Advanced Nursing, 66(1), 22-32. https://doi.org/10.1111/ j.1365-2648.2009.05190.x

Varpio, L., Ajjawi, R., Monrouxe, L. V., O’Brien, B. C., \& Rees, C. E. (2017). Shedding the cobra effect: Problematising thematic emergence, triangulation, saturation and member checking. Medical Education, 51(1), 40-50. https://doi.org/10.1111/ medu.13124

Varpio, L., Paradis, E., Uijtdehaage, S., \& Young, M. (2020). The distinctions between theory, theoretical framework, and conceptual framework. Academic Medicine, 95(7), 989-994. https://doi.org/10.1097/ACM.0000000000003075

Wainwright, S. F., Shepard, K. F., Harman, L. B., \& Stephens, J. (2011). Factors that influence the clinical decision making of novice and experienced physical therapists. Physical Therapy, 91, 87-101. https://doi.org/10.2522/ptj.20100161

Williams, V., Kinnear, D., \& Victor, C. (2016). "It's the little thing that count": Healthcare professionals' views on delivering dignified care: A qualitative study. Journal of Advanced Nursing, 72(4), 782-790. https://doi.org/10.1111/jan.12878

Xavier, K., Shepherd, L., \& Goldstein, D. (2007). Clinical supervision and education via videoconference: A feasibility project. Journal of Telemedicine and Telecare, 13(4), 206-209. https://doi.org/10.1258/135763307780907996

Yoon, L., Campbell, T., Bellemore, W., Ghawi, N., Lai, P., Desveaux, L., Quesnel, M., \& Brooks, D. (2017). Exploring mentorship from the perspective of physiotherapy mentors in Canada. Physiotherapy Canada, 69(1), 38-46. https://doi.org/10.3138/ ptc. $2015-52$ 